\title{
Propuesta de criterios para la evaluación de la investigación española en biblioteconomía y documentación: la producción de los científicos y de los centros de investigación
}

\author{
JosÉ LóPEZ YEPES \\ Universidad Complutense de Madrid (España) Tel. (3491) 3-94-20-55 \\ E-mail: yepes@ ccinf.ucm.es \\ Judith Prat Sedeño \\ Universitat de Lleida (España)
}

\section{RESUMEN}

El artículo presenta una relación de posibles criterios para diagnosticar y valorar la investigación española en materia de biblioteconomía y documentación, entre los cuales se encuentran el interés por esta problemática en nuestra área de conocimientos; la producción y el factor de impacto de la publicaciones científicas; la formación de los investigadores; las tendencias temáticas; la tipología de los métodos de investigación utilizados; los focos de investigación y las escuelas científicas; el impacto de la investigación en la comunidad profesional; la producción y el prestigio de los científicos y de los centros de investigación; la repercusión de la investigación en el ámbito de la ciencia española, y el grado recíproco de repercusión de la ciencia extranjera y de la ciencia española en este sector de conocimientos. Se pone especial énfasis en el estudio de la repercusión de los autores españoles y extranjeros sobre la base de las citas recibidas y analizadas en una base de datos mantenida desde 1985 por la Facultad de Biblioteconomía y D ocumentación de la Universidad de G ranada (España).

Palabras Clave: Investigación bibliotecológica española; Investigación científica;

Evaluacion de proyectos; Citas bibliográficas.

\section{CRITERIA PROPOSAL FOR THE ASSESSMENT OF SPANISH RESEARCH IN LIBRARY SCIENCE AND DOCUMENTATION: THE PRODUCTION OF SCIENTISTS AND RESEARCHS CENTERS}

\author{
JoSÉ LóPEZ-YEPES \\ Judith Prat-Sedeño
}

\begin{abstract}
This paper proposes a set of criteria to be used in assessing and evaluating Spanish research in Library and Information Science. Such criteria includes current interest for this problematic in our field, production and influence of published research, formation of researchers, thematic concerns, research methods employed, research foci and schools, the impact
\end{abstract}

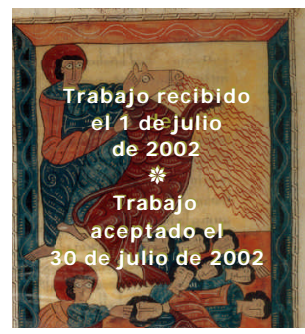


of research in the professional community and the effect on Spanish research of work done abroad and vice-versa. Special attention is given to analyzing the prestige of Spanish and international authors on the basis of references to their work recorded in a database created by the University of $\mathrm{G}$ ranada's $\mathrm{Fa}$ culty of Library and Information Science (Spain).

Palabras Clave: Spanish science research; Scientific research; Projetc evaluation; Bibliographic citations.

\title{
INTRODUCCIÓN
}

\begin{abstract}
iortamente la década prodigiosa de los años 90 ha presenciado en España numerosos logros para nuestro campo de conocimientos. Junto a la implantación de la licenciatura y el doctorado en documentación, la creación de nuevas revistas científicas, el gran crecimiento de centros y profesorado para estas enseñanzas, debemos sumar el aumento delainvestigación del sector cristalizado en un número creciente de tesis doctorales, en la publicación de colecciones de monografías, en la edición de artículos y actas de congresos, etcétera, pero sobre todo, en la aparición, desde 1998, de trabajos que tratan de evaluar y establecer diagnósticos acerca de nuestra investigación, sus tendencias, sus logros, su papel, en suma, en el ámbito del contexto de la documentación española en la ciencia universal. Son muestras de lo que digo, los trabajos sobre producción, tendencias, frentes de investigación, análisis de citas, etcétera de D elgado López-Cózar, autor del único y más reciente estudio de conjunto ${ }^{1}$ y precedidos por otros de la escuela granadina como Moya, ${ }^{2}$ Jiménez Contreras, ${ }^{3}$ etcétera, además de Frías ${ }^{4}$ y otras investigaciones a nivel de tesis doctoral. ${ }^{5}$
\end{abstract}

1 - Emilio D elgado López-Cózar. "Diagnóstico de la investigación en Biblioteconomía y D ocumentación en España"

- Emilio D elgado López-Cózar. "La investigación en Biblioteconomía y D ocumentación"

- Emilio D elgado López-Cózar. "Las revistas españolas de Ciencias de la D ocumentación, productos manifiestamente mejorables"

2 - Félix de MoyaA negón; López Gijón, J. y García Caro, C. TénicasarantitativasaplicadasalaBiblide conomáayla Doumentacón

- Félix de Moya Anegón y Jiménez Contreras, E. "Research Fronts in Library and Information Science in Spain"

- Félix de Moya Anegón y Jiménez Contreras, Evaristo. "Autores españoles más citados en Biblioteconomía y D ocumentación"

- Félix de Moya Anegón y Herrero Solana, Víctor. "Visibilidad internacional de la producción científica iberoamericana en Biblioteconomía y D ocumentación"

- Félix de Moya Anegón. "La investigación española en Recuperación de la Información: Análisis bibliométrico (1984-1999)"

3 Evaristo Jiménez Contreras y Moya Anegón, Félix de. "Análisis de la autoría en revistas españolas de Biblioteconomía y D ocumentación".

4 J. A. Frías y Romero, P. “¿Quienes son y qué citan los investigadores que publican en las revistas españolas de Biblioteconomía y D ocumentación?"

5 - Rosario Arquero Avilés. La produrción dentífica en Biblideconmáay Doumentadón en Espeña - Ángel Borrego Huerta. "La investigación cualitativay sus aplicaciones en Biblioteconomía y D ocumentación" 
En las líneas que siguen, fruto de una primera pero esperanzadora reflexión, nos proponemos presentar algunos criterios - un posible decálogo- que podrían permitir diagnosticar y evaluar la actividad investigadora de la ByD en España, bien desde una perspectiva personal como desde una institucional; y especialmente el papel que desempeñan los científicos de modo individual y en el ámbito de sus centros de trabajo. Los criterios a los que nos referimos son los siguientes:

1. Interés de nuestra comunidad científica por la problemática de la investigación en ByD.

2. Producción y factor de impacto de las publicaciones científicas.

3. Formación de investigadores.

4. Las tendencias temáticas.

5. El uso de métodos variados de investigación.

6. Los motores del crecimiento de las investigaciones y su difusión a nivel nacional e internacional.

7. El impacto de la investigación en la comunidad profesional.

8. La productividad y el impacto de los científicos y de los centros universitarios.

9. La repercusión y reconocimiento de nuestra investigación en el ámbito de la ciencia y de la política científica en España.

10. El grado recíproco de influencia de la investigación española y extranjera en ByD.

\section{INTERÉ S DE NUEST RA COMUNID AD CIENTÍFICA POR LA PROBLEMÁTICA DE LA IN VESTIGACIÓN EN BYD}

Es evidentela preocupación cada vez mayor de nuestro colectivo por los problemas de indagación. D esde la feliz presencia de materias troncales de formación de investigadores en los planes de estudio de la licenciatura y el doctorado, debemos tener en cuenta algunos otros hitos como: 1) los trabajos referenciados al principio de este texto; 2) la celebración de jornadas de estudio como la citada al principio, la celebrada sobre comunicación científica en la Universidad de Salamanca en febrero pasado o las primeras celebradas en 1996 en las Universidades de Barcelonay de Zaragoza; ${ }^{6}$ 3) la recopilación de citas de autor emprendida por la Universidad de G ranada desde el periodo 1985 hasta nuestros días o la tesis doctoral sobre producción científica de nuestro sector desde 1975 hasta 1984, debida a la profesora Charo Arquero de la Universidad Complutense de Madrid, ${ }^{7}$ y 4) Monografías en curso de publicación como un Manual demítodos aualitativosparaByD en la Editorial Síntesis, de Madrid.

6 Jesús Tramullas Saz. Tendenias delainvestigaiónenDœumetaaión Zaragoza

7 Rosario Arquero Avilés. Op Cit. 


\section{PRODUCCIÓN Y FACTOR DE IMPACTO DE LAS PUBLICACIONES CIENTÍFICAS}

Medirlaproducción delasinvestigaciones y el valor de dichaproducción son, bá sicamente, los objetivos de este criterio. Sobre ello, procede, en mi opinión, establecer los siguientes principios: 1) ¿Meras publicaciones o auténticos trabajos de investigación? No es nuestra disciplina la única aquejada de una gran parte de trabajos publicados que aportan poco o que sólo representan la descripción de fenómenos sin alcance universal en muchos casos. Los responsables de las revistas y demás medios deberían exigir para la publicación que los trabajos presentaran un problema científico objetivo, es decir, no resuelto; justificaran el método a emplear; ejecutaran lanecesaria reflexión sobre las fuentesy alcanzaran unas conclusiones finales o soluciones al problema planteado al principio. 2) La producción de tesis doctorales en nuestra materia es creciente como numerosos son los focos de producción: facultades de biblioteconomía y documentación, facultades de ciencias de la información, facultades de filosofíay letras, facultades de medicina y otras facultades susceptibles de presenciar la realización de una tesis doctoral de documentación aplicada a una disciplina concreta. Según Jiménez Contreras se presentaron 270 tesis doctorales de las cuales se han leído 61, 60, 33y 24 en las Universidades deValencia, Complutense, Murcia y $\mathrm{G}$ ranada respectivamente. Los doctores en documentación son muy recientes dada la juventud de la carrera. El primer doctor en documentación - previamente diplomado y licenciado en documentación- lo fue en la Universidad de G ranada en el año 2002. 3) La producción de monografías tiene su origen, principalmente, en las tesis doctorales y en los proyectos docentes para ocupar plazas de profesorado y, como se sabe, hay, al menos, dos editoriales, Síntesis, en Madrid, y Trea, en G ijón, que mantienen colecciones de estaíndole. 4) La calidad delos artículos de las revistas es directamente proporcional al impacto de las revistas que los albergan. Son numerosas las revistas de ByD , aunque la mayor parte son editadas por asociaciones profesionales y Universidades. La de mayor impacto - RexistaEspa ñdadeDoumentaciónCientífica- es editada, por el contrario, por el CIND o C (Consejo Superior de Investigaciones Científicas). Recientemente, D elgado López-Cózar ${ }^{8}$ ha señalado las condiciones para mejorar la calidad de nuestras revistas y, en consecuencia, puedan ser recogidas en los índices de citas internacionales. Adjuntamos la lista de publicaciones periódicas españolas por orden del factor de su impacto, según el estudio del autor acabado de mencionar:

1. Revista Españda deDoumentación Científica (CSIC-CIND O C), 1,57

8 Emilio D elgado López-Cózar. "Las revistas españolas de Ciencias..." 
2. BdéńndeANABAD (A sociación Nacional de Archiveros, Bibliotecarios y D ocumentalistas), 1,28

3. Doumetadóndelas CieniasdelaInfomacón(D epartamento de Biblioteconomía y D ocumentación, U. Complutense de Madrid), 1,03

4. El Profesional dela Informacón(Swets), 1,03

5. Jomadas Catalamas deDoumentación (Sociedad Catalana de D ocumentación), 0,81

Por lo que se refiere a productividad, Frías y Romero ${ }^{9}$ han establecido en 1998 el siguiente elenco:

1. Bdedín delaANABAD 15,9\%

2. ReistaGeneal deInfomadónyDoumentacoón(D epartamento de Biblioteconomía y D ocumentación, Universidad Complutense de Madrid), 9,3 \%

3. ReistaEspañda deDcumentaciónCientífica (CSIC-CIND OC) 8,9 \%

4. Bdeín dela AscaiaciónAndaluza deBiblictecarios 7,7 \%

\section{FORMACIÓN DE INVEST IGADORES}

Hemos indicado antes que nuestros estudiantes se forman reglamentariamente en las técnicas de investigación, de modo optativo, en varias diplomaturas, y de modo obligatorio en las licenciaturas. La formación de los investigadores en ByD comporta el examen de los siguientes aspectos: 1) Los programas de doctorado incluyen una asignatura de metodología de la investigación. Sería conveniente un estudio crítico de los mismos que evaluara el contenido global del programa y, a estos efectos, el contenido y los instrumentos didácticos de que se sirven los cursos de carácter metodológico. 2) Parece necesaria la existencia de manuales de formación de investigadores en ByD así como de métodos cualitativos y cuantitativos específicos de nuestro campo.10 3) La elección de un buen director es, como se sabe, la pieza fundamental dela formación. Las condiciones que debe reunir un buen director son difíciles de conjugar en una misma persona con el agravante de que el investigador queno seforma se malformay se convierte subsiguientemente en un mal director. ${ }^{11}$

En nuestra opinión, en los últimos tiempos la calidad de las tesis doctorales no se mantiene, en general, en el nivel deseable o superable. Las causas son diversas: la urgencia con la que algunos profesores desean doctorarse a fin de concurrir a plazas de

9 J. A. Frías y Romero, P. Op Cit

10 - Ángel Borrego Huerta. "La investigación cualitativa y sus aplicaciones en Biblioteconomía y Documentación".

- Borrego Huerta, Angel. Méddogáa aralitativa deinvestigadónenBiblideconomáy Doumetaaón

11 - José M. D esantes G uantery López Y epes, José. Teoríay técnica delainvestigación científica.

- José López Y epes. Laaventura dela investigación científica. G uía del investigadory del director de investigación.

- José López Y epes. Loscaminosdelainformacón Cómobuscar, sdecionaryagganizarnustradbametaaón pesonal. 
profesorado estable; la falta de competitividad a la hora de alcanzar la máxima calificación en el acto académico de presentación y defensa de la tesis; la escasez de cursos de metodología de la investigación, y la escasa consulta de manuales de la misma materia. Sin embargo, resta por mencionar otra causa que, en último término, es la raíz de las anteriores. Se trata de la figura del director de la investigación, auténtico formador de los doctorandos, y responsable definitivo de la trayectoria futura delos anteriores. G eneralmente, se ha prestado atención a la formación del futuro investigador, pero ¿cómo se forma el director de la tesis doctoral? Hasta el momento, se forma siguiendo los pasos y el ejemplo de su director de tesis y así sucesivamente, pero sólo en la medida en que dicho director haya actuado con total competencia y responsabilidad. En caso contrario, sería necesario arbitrar una serie de medidas que garantizaran la competencia de los directores de tesis doctorales de acuerdo con las normas de rigor y seriedad que deben guiar toda actividad de carácter científico. ${ }^{12}$

\section{LASTEN DENCIASTEMÁTICAS}

La elección de tema de investigación en nuestras disciplinas se plantea, al menos, en dos circunstancias: en la que interviene el investigador en formación o doctorando, y en la que interviene el investigador ya consagrado y con determinada experiencia. Los temas de tesis doctorales deben reunir suficientes requisitos para que el investigador se forme de modo integral y profundo, y lleve las peculiaridades del tema hasta las últimas consecuencias. Por el contrario, el investigador consagrado se puede permitir el lujo de intentar aproximaciones o de ensayar nuevos caminos. En todo caso, los temas pueden representar problemas inherentes a la sociedad en la que se desenvuelve el investigador o problemas de mayor calado susceptibles de afectar a un mayor número de destinatarios en el ámbito de la ciencia universal. Los estudios sobre tendencia temática y caracterización de frentes de investigación se iniciaron, según nuestros datos, con el trabajo de Moya y colaboradores ${ }^{13}$ y confluyen con el trabajo sobre diagnóstico de D elgado López-Cózar ${ }^{14}$ y otros dedicados al estudio de la temática de las tesis doctorales en el departamento de ByD de la Universidad Complutense de Madrid. ${ }^{15}$ Como resumen, indiquemos que, para el periodo 1977-1994, el profesor Jiménez Contreras establece el siguiente cuadro temático:

$\begin{array}{lr}\text { Profesión } & 5,9 \% \\ \text { Historia del libro y las bibliotecas } & 0,8 \% \\ \text { Servicios en bibliotecas 1 } & 9,5 \% \\ \text { Almacenamiento y recuperación } & 18,5 \%\end{array}$

12 José M. D esantes Guanter y López Y epes, José. Op Cit.

13 Félix de Moya Anegón y Jiménez Contreras, Evaristo. "Autores españoles más citados..."

14 Emilio Delgado López-Cózar. "Diagnóstico de la investigación..."

15 José López Y epes. "Focos de investigación y escuelas científicas en D ocumentación. La experiencia de las tesis doctorales". 


$\begin{array}{lr}\text { Búsquedas de información } & 7,3 \% \\ \text { Comunicación 1 } & 8,3 \% \\ \text { Teoría } & 4,6 \% \\ \text { Otros } & 14,1 \%\end{array}$

\section{EL USO DE MÉTODOSVARIADOS DE INVESTIGACIÓN}

La diversidad de métodos de investigación y su aplicación a una amplia temática son un índice de madurez en los procesos de investigación de un determinado campo del saber. En E spaña todavía adolecemos de ambos componentes. D e entrada, contamos con metodologías de carácter cuantitativo ${ }^{16}$ y una reciente tesis doctoral dedicada a los métodos cualitativos, ${ }^{17}$ avance de un futuro manual en preparación con la colaboración del profesor Frías. Por su parte, D elgado López-Cózar advierte en la actualidad cierta variabilidad metodológica aunque se utilizan pocos métodos cualitativos como la encuesta y, en cambio, predomina el análisis de contenido y los métodos bibliométricos. ${ }^{18} \mathrm{E}$ n todo caso, es evidente la necesidad de que nos abramos al campo de la multiplicidad de los métodos y al diseño de otros nuevos más allá, si es posible, de la tradicional dicotomía cualitativo/ cuantitativo.

\section{LOS MOTORES DEL CRECIMIENTO DE LAS IN VESTIGACIONESY SU DIFU - SIÓN}

Bajo este epígrafe, comprendemos la valoración de aquellos factores que hacen posible que se inicien las investigaciones, que se establezcan relaciones de carácter científico-profesional y que, finalmente, el fruto de todo ello pueda ser difundido a la comunidad científica nacional e internacional del modo más eficaz. Pensemos, asimismo, que ello constituirá un elemento más que hará posible la evaluación de la ciencia documental y de sus científicos y que será la base para una determinada política en el sector. En un trabajo reciente, hemos estudiado la formación y difusión de la investigación en el Departamento de Biblioteconomía y Documentación de la Universidad Complutense de Madrid (algo que desearíamos hacer extensible al resto de las universidades) obteniendo datos acerca de a) el número y los títulos de las tesis doctorales leídas; b) la productividad delos directores; c) los focos de investigación; es decir, aquellos profesores o instituciones que inician y desarrollan líneas de investigación a través de los temas de las tesis dirigidas; c) las escuelas científicas, constituidas por generaciones de científicos que de modo sucesivo van dirigiendo tesis agrupándose en genealogías y, finalmente, d) el grado de influenciainstitucional

16 Félix de Moya Anegón; López Gijón, J. y G arcía Caro, C. Ténicas arantitativas..

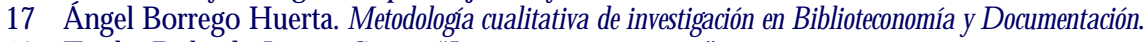

18 Emilio Delgado López-Cózar. "La investigación en..." 
que se va estableciendo entre las universidades según la propagación de las ideas representadas por los directores. ${ }^{19}$

En cuanto a la difusión de nuestras investigaciones, y más allá del recurso a los medios como las monografías y las revistas en soporte papel o electrónico, no parece que se nos reconozca demasiado prestigio en la comunidad internacional salvo en la iberoamericana. A solucionar esta laguna se encamina la revista Jaumal of Reserch onInformation Saience/ Reista Iberoameicana deInvetigacón en Cienia dela Infomacióny Doamentadón, que hace dos años comenzó a editar nuestro departamento. En esta revista, muy vinculada a las universidades iberoamericanas y con un Consejo E ditorial que incluye a profesores norteamericanos e iberoamericanos, deberían participar también otras universidades españolas e iberoamericanas pues, como se sabe, los problemas de la investigación nos afectan a todos sin excepción alguna.

\section{EL IMPACTO DE LA IN VESTIGACIÓN EN LA COMUNIDAD PROFESIONAL}

Se ha señalado como punto débil del estado de nuestra investigación y como prueba de inmadurez, la existencia de un frente bibliotecario disociado, en mayor o menor medida, del frente universitario. Las causas son lejanas en el tiempo pero también es verdad que se van aminorando con el tiempo. La celebración de congresos organizados por asociaciones profesionales a los que acudimos los universitarios, la vinculación de temas de tesis doctorales a problemas cotidianos observados por los profesionales, la relación cada vez mayor entre docentes y bibliotecarios de las universidades en el desarrollo de nuestras enseñanzas, la reciente inclusión delos títulos de tesis doctorales leidas en dos universidades madrileñas en las páginas CLIP, BdedíndelaSedic(Sociedad Española de D ocumentación e Información Científica), son muestras inequívocas de un evidente acercamiento.

\section{LA PRODUCTIVIDAD Y EL IMPACTO DE LOS CIENTÍFICOSY DE LOSCEN- TROS UNIVERSITARIOS}

Un indicador de gran importancia es, sin duda, lainfluencia delas ideas científicas obtenidas en las investigaciones de los profesores universitarios y, en consecuencia, su repercusión no sólo a nivel personal sino a nivel institucional. D esconociéndose los tramos de investigación que poseen los profesores universitarios (recompensa económica que pueden recibir los profesores cuando someten su investigación a evaluación objetiva en periodos de seis años), la base de partida la ha constituido la lista de autores citados - españoles y extranjeros- con más de 10 citas en el periodo 1985-2002 en la base de datos que mantiene actualizado el equipo de trabajo comandado por el profesor Moya, de la Universidad de Granada, a quien agradecemos

19 José López Yepes. "Focos de investigación..." 
públicamente su generosa ayuda. A un reconociendo las deficiencias que presentan estas aproximaciones por su carácter mecánico y cuantitativo, por el origen de algunas citas debidas a determinados convencionalismos 0 artificios y por la mayor 0 menor antigüedad en el tiempo investigador y el número de miembros de los distintos departamentos, creemos que merece la pena conocer estos datos y obtener las conclusiones o aplicaciones que se consideren oportunas. Sin duda, un estudio posterior debería tratar de analizar las tendencias investigadoras e, incluso, las situaciones personales de los investigadores en su ámbito de actuación. También resultaría muy conveniente determinar qué departamentos imparten programas de doctorado en universidades extranjeras. En relación con el primer aspecto, se ordenan dichos organismos del modo que sigue:

\section{Relación de investigadores y departamentos por número de citas recibi- das según la Base de D atos de la U . de G ranada (1985-mayo 2002)}

\section{U niversidad C omplutense de M adrid}

Nombres

López Y epes, J.,

Sagredo Fernández, F.,

López Y epes, A.,

Valle Gastaminza, F. del,

Costa Carballo, C. M. da,

Bernal Cruz, F.J.,

Peón, J.,

Espinosa Temiño, Blanca.,

Maciá, M.,

Ramos Simón, L.F.,

López López, P.,

Tejada, C.,

Tdales

№ de investigadores:

№ de citas:
№ de citas

208

86

59

31

30

27

20

15

13

12

11

11

12

523

\section{U niversidad de Valencia. E studio G eneral}

Nombres

Terrada Ferrandis, María Luz,

López Piñero, J.M.,
No de citas

185

167 
Cueva, A. de,

Aleixandre, R.

21

O sca, Julia

Tdales

№ de investigadores:

№ de citas:

\section{U niversidad de G ranada}

Nombres № de citas

Moya Anegón, F. de,

Pinto Molina, María,

Hípola, P.,

D elgado López-Cózar, E.,

Jiménez Contreras, E.,

Torres, Isabel de,

Ruiz Pérez, R., 24

López Gijón,

Ruiz Rodríguez, A.A.,

Fernández Molina, J.C.,

Molina, E. (+),

Moneda, M. de la,

Herrero Solana, V.F.,

Totales

$\mathrm{N} N$ de investigadores:

№ de citas:

\section{U niversidad de B arcelona}

Nombres № de citas

Amat Noguera, Nuria,

Abadal Falgueras, E.,

Mayol, Carme,

Massissimo, Angels,

Mañá, Teresa

Espelt, Constanza,

Pons Serra, A.

Sebastiá, Montserrat,

Miralpeix, Concepció, 
№ de investigadores:

№ de citas:

U niversidad C arlos III (M adrid)

Nombres № de citas

Sanz Casado, Elías, 72

Caridad, Mercedes 61

Moreiro, José Antonio 44

O rtiz-Repiso, Virginia 30

López de Quintana, E.,

Tdales

№ de investigadores:

№ de citas:

\section{U niversidad de M urcia}

\section{Nombres}

№ de citas

Izquierdo, José María

Ros García, Juan

Méndez, F.J.,

Rodríguez Muñoz, José V.

Gómez Hernández, J.A.

Saorín Pérez, T.

Tdales

№ de investigadores:

№ de citas:

\section{U niversidad A utónoma de B arcelona}

Nombres

Fuentes i, M.E.,

Coll Vinent, R.,

Recoder, M.J.,

Totales

№ de investigadores:

№ de citas:

\section{No de citas}

73

57

37

3

167 
U niversidad A utónoma de M adrid

Nombres № de citas

Currás, Emilia, 153

Totales

№ de investigadores:

№ de citas:

U niversidad de Salamanca

Nombres № de citas

Merlo, J.A., 36

Sorli, Angela, 31

Cordón, J.A.

Frías, J.A.,

García Caro, Concepción,

Tdates

№ de investigadores:

№ de citas:

U niversidad Pompeu Fabra (B arcelona)

Nombres № de citas

Codina Bonilla, Ll.,

Totales

№ de investigadores:

№ de citas:

U niversidad de A Icalá

Nombres

Moscoso, Purificación,

Zulueta, M. Ángeles,

№ de citas

70

28

Totales

№ de investigadores:

№ de citas:

U niversidad de Sevilla

Nombres № de citas García Gutiérrez, A.L., 
Totales

№ de investigadores:

№ de citas:

U niversidad de Zaragoza

Nombres № de citas

García Marco, F.J.,

Tramullas, J.,

Totales

№ de investigadores:

№ de citas:

U niversidad de Extremadura

Nombres № de citas

Castro Castro, C.,

Pulgarín, Antonio

Muñoz Cañavate, A.,

Tdales

№ de investigadores:

№ de citas:

U niversidad San Pablo-C E U (M adrid)

Nombres № de citas

Galdón, Gabriel, 28

Tdales

№ de investigadores:

№ de citas:

\section{U niversidad de L a C oruña}

Nombres № de citas

Fuentes Romero, J.J.,

Tdales

№ de investigadores:

№ de citas: 


\section{U niversidad de la Laguna}

Nombres

González Antón, F.J. № de citas

Tdales

№ de investigadores

24

№ de citas:

\section{CSIC (C indoc)}

Nombres

Gómez Caridad, M. Isabel,

Méndez Miaja, Aida,

Pérez Alvarez-O ssorio, J.R.,

Vázquez, Manuela,

Ferreiro, L.,

Román, Adelaida,

Viesca, Rosa de la

Aguillo, Isidro,

Sancho, Rosa,

Galbán, Carmen,

Villagrá, Angel,

Valle, Antonio,

Alcaín, D olores,

Irazazábal, Amelia,

Lara, Alfredo,

Rey, Alfredo del,

Tdales

No de investigadores:

№ de citas:
№ de citas

147

115

113

88

38

35

33

33

31

28

28

27

21

21

19

15

16

792

\section{Relación de departamentos universitarios por número de investigadores citados con más de 10 citas ( 1985-mayo 2002)}

1. Universidad de $\mathrm{G}$ ranada

2. Universidad Complutense de Madrid 12

3. Universidad de Barcelona 9

4. Universidad de Murcia 6

5. Universidad de Valencia. Estudio General 5

5. Universidad Carlos III 5

5. Universidad de Salamanca 5

6. Universidad Autónoma de Barcelona 3 
6. Universidad de Extremadura 3

7. Universidad de Alcalá 2

7. Universidad de Zaragoza 2

8. Universidad Autónoma de Madrid 1

8. Universidad Pompeu Fabra 1

8. Universidad de Sevilla 1

8. Universidad San Pablo-CEU 1

8. Universidad de La Coruña 1

8. Universidad de La Laguna 1

- CSIC (Cindoc) 16

Relación de departamentos universitarios por número de citas recibidas ( 1985-mayo 2002)

1. Universidad Complutense de Madrid 523

2. Universidad de Valencia. Estudio G eneral 421

3. Universidad de Granada 380

4. Universidad de Barcelona 338

5. Universidad Carlos III 218

6. Universidad de Murcia 168

7. Universidad Autónoma de Barcelona 167

8. Universidad Autónoma de Madrid 153

9. Universidad de Salamanca 112

10. Universidad Pompeu Fabra 103

11. Universidad de Alcalá $\quad 98$

12. Universidad de Sevilla 73

13. Universidad de Zaragoza 37

14. Universidad de Extremadura 35

15. Universidad San Pablo-CEU 28

16. Universidad de La Coruña 26

17. Universidad de La Laguna 24

- CSIC (Cindoc) 792

Relación de departamentos según su miembro más citado y número de citas correspondientes ( 1985-mayo 2002)

1. López Yepes, José Universidad Complutense de Madrid 208

2. Terrada Ferrandis, M. Luz Universidad de Valencia. Est. gral. 185

3. Puente, Emilia Universidad Autónoma de Madrid 153

4. Codina Bonilla, Lluis Universidad Pompeu Fabra 103

5. Amat Noguera, Nuria Universidad de Barcelona 96

6. Izquierdo Arroyo, J.M. Universidad de Murcia 74

7. G arcía G utiérrez, A. L. Universidad de Sevilla 73 
8. Fuentes i , M. Eulalia Universidad Autónoma de Barcelona

73

9. Sanz Casado, Elías Universidad Carlos III

72

10. Moscoso Castro, Purificación Universidad de Alcalá

70

11. Moya Anegón, Félix de Universidad de Granada

63

12. Merlo Vega, José A. Universidad de Salamanca

36

13. Galdón López, Gabriel Universidad San Pablo-CEU

28

14. Fuentes Romero, Juan J. Universidad de La Coruña

26

15. G onzález Antón, F.J. Universidad de La Laguna

24

16. García Marco, F. J. Universidad de Zaragoza

21

17. Castro Castro, Carlos Universidad de Extremadura

13

\section{REPERCUSIÓN DE LA INVESTIGACIÓN DOCUMENTAL EN EL ÁMBITO DE LA CIENCIA Y DE LA POLÍTICA CIENTIÍFICA EN E SPAÑ A}

En este último criterio se trataría de conocer cuál es la actitud y el posible reconocimiento de los científicos españoles de cualquier rama del saber en relación con el cumplimiento de las tres funciones básicas que se atribuyen a la documentación, a saber: la función de apoyo al crecimiento de los saberes, la función de apoyo a la difusión de los hallazgos científicos y la función de evaluación de la actividad científicas, ${ }^{20}$ así como el uso de métodos propiamente documentales en otras áreas del saber. Consecuentemente, ello tendría la adecuada repercusión en el ámbito de la política científica.

\section{EL GRADO RECÍPROCO DE INFLUEN CIA DE LA IN VESTIGACIÓN ESPAÑO- LA YEXTRANJERA EN BYD}

Launiversalización de lainvestigación española en ByD exige como objeto de estudio, de un lado, conocer lainfluencia sobre nosotros de investigadores extranjeros y, de otro, la repercusión de nuestras ideas dadas a conocer en publicaciones extranjeras. Para el primer aspecto, sigue siendo de eficaz ayuda la tan comentada Base de D atos de citas de la Universidad de Granada. D el conjunto del listado de autores con 10 citas al menos, se extrae que el grupo universitario y del Consejo Superior de Investigaciones Científicas (Cindoc) ocupa un 20\% de los autores a los que corresponde el 30\% aproximadamente de citas. Frente a ello, el sector profesional español ocupa el 30\% de autores y también un 30\% de citas. Sin embargo el grupo de extranjeros es todavía muy notable: el $50 \%$ de autores y nada menos que el $40 \%$ de citas. La colonización es, pues, muy notable. Sin embargo, estas cifras se aminoran a nuestro

20 José López Yepes. “La evaluación de la ciencia en el contexto de las Ciencias de la... D ocumentación". 
favor si contemplamos los porcentajes en la lista de autores que reciben al menos 50 citas. En este caso, el grupo de profesores universitarios y de investigadores del Consejo alcanza un porcentaje del 58\% y un número de citas cifrado en el $62 \%$ aproximadamente. Se trata, pues, de un núcleo de gran importancia frente al grupo de autores extranjeros que, en idéntica circunstancia, se limita al $23 \%$ tanto en número de autores como de citas.

Porcentajes según número de investigadores universitarios, del C SIC y el resto de investigadores (profesionales) españoles y extranjeros con más de 10 citas y número total de citas ( 1985-mayo 2002)

\begin{tabular}{|l|c|c|c|c|}
\hline & No de investigadores & \% & No de citas & \% \\
\hline Universidad & 71 & 15.57 & 2886 & 23.65 \\
\hline CSIC (Cindoc) & 16 & 3.51 & 744 & 6.10 \\
\hline Resto (Españoles) & 139 & 30.48 & 3686 & 30.21 \\
\hline Resto (Extranjeros) & 230 & 50.44 & 4885 & 40.04 \\
\hline Total & 456 & 100 & 12,201 & 100 \\
\hline
\end{tabular}

\section{Lista de autores españoles y extranjeros que han recibido, al menos, 50 citas ( 1985-mayo 2002)}

\begin{tabular}{|l|c|l|}
\hline 1. López Yepes, José & 208 & Universidad \\
\hline 2. Terrada Ferrandis, María & 185 & Universidad \\
\hline 3. Garfield, E., & 172 & \\
\hline 4. López Piñero, J.M. & 167 & Universidad \\
\hline 5. Currás, Emilia & 153 & Universidad \\
\hline 6. Gómez Caridad, M.Isabel & 147 & CSIC \\
\hline 7. Lancaster, F.W. & 146 & \\
\hline 8. Méndez Miaja, Aida & 115 & CSIC \\
\hline
\end{tabular}




\begin{tabular}{|c|c|c|}
\hline 9. Pérez Alvares-O ssorio, J.R. & 113 & CSIC \\
\hline 10. Bonilla Codina, Lluis & 103 & Universidad \\
\hline 11. Amat Noguera, Nuria & 96 & Universidad \\
\hline 12. Agenjo Bullón, Xavier & 94 & Profesional \\
\hline 13. Price, D. de Solla & 92 & \\
\hline 14. Line, Maurice & 88 & \\
\hline 15. Sagredo Férnandez, Félix & 88 & Universidad \\
\hline 16. Vázquez Valero, Manuela & 88 & CSIC \\
\hline 17. Anglada i de Ferrer, Lluis & 84 & Profesional \\
\hline 18. Carrión Gútiez, Manuel & 78 & Profesional \\
\hline 19. Izquierdo Arroyo, J.M. & 74 & Universidad \\
\hline 20. Fuentes i Pujol, M. Eulalia & 73 & Universidad \\
\hline 21. G arcía Gutiérrez, Antonio L. & 73 & Universidad \\
\hline 22. Sanz Casado, Elías & 72 & Universidad \\
\hline 23. Moscoso Castro, Purificación & 70 & Universidad \\
\hline 24. Cornella, Alfons & 68 & $\begin{array}{l}\text { Profesional- } \\
\text { Universidad }\end{array}$ \\
\hline 25. D esantes-Guanter, J.M. & 66 & Universidad \\
\hline 26. Estivill i Rius, Assumpció & 66 & Universidad \\
\hline 27. Moya Anegón, Félix de & 63 & Universidad \\
\hline 28. Caridad Sebastián, Mercedes & 61 & Universidad \\
\hline 29. Cronin, B. & 61 & \\
\hline 30. Brookes, B.C. & 60 & \\
\hline 31. Abadal Falgueras, E. & 60 & Universidad \\
\hline 32. López Y epes, Alfonso & 59 & Universidad \\
\hline
\end{tabular}




\begin{tabular}{|l|c|l|}
\hline 33. Slype, G eorges van & 59 & Universidad \\
\hline 34. Mayol i Fernández, Carme & 59 & \\
\hline 35. Salton, Gérard & 57 & \\
\hline 36. Coll Vinent, R. & 57 & Universidad \\
\hline 37. Narin, F. & 56 & \\
\hline 38. Pinto Molina, María & 54 & Universidad \\
\hline 39. Schubert, A. & 54 & \\
\hline 40. Fernández Muñoz, M. Teresa & 52 & Profesional \\
\hline 41. Bordons, M. & 52 & Profesional \\
\hline 42. Camí, J. & 51 & Profesional \\
\hline 44. Guardiola, E. & 50 & Profesional \\
\hline
\end{tabular}

\section{Porcentajes de presencia de autores españoles y extranjeros con 50 citas (1985-mayo 2002)}

\begin{tabular}{|l|c|c|c|c|}
\hline \multicolumn{1}{|c|}{ Tipología } & No & \% & No de citas & \% \\
\hline Profesores Univ. & 21 & 47.72 & 1845 & 49.49 \\
\hline Investigadores CSIC & 4 & 9.10 & 463 & 12.41 \\
\hline Profesionales españ. & 9 & 20.45 & 578 & 15.50 \\
\hline Profes.y prof. extranj. & 10 & 22.73 & 842 & 22.58 \\
\hline Totales & 44 & 100 & 3728 & 100 \\
\hline
\end{tabular}

La repercusión de la investigación española en el extranjero se mide, entre otros extremos, observando qué personas y qué instituciones publican, en qué revistas, quétemas y cuál es la producción frente a otros países. En la reciente II Jomada de recerca celebrada en Barcelona (19 de junio de 2002), el profesor Evaristo Jiménez Contreras ofrecía una serie de datos de sumo interés: 
A) La investigación española en el exterior es, fundamentalmente, académica siendo sus focos la universidad y el CSIC (más del $80 \%$ ) sin presencia de las instituciones bibliotecarias.

B) Los autores más productivos son Gómez Caridad, Isabel (9), Méndez, Aida (9), Campanario, J.M. (6), Currás, Emilia (6), Bordons, María (6), Pulgarín, Antonio (6), Plaza, Luis M. (5), Alvarez, P. (5), Fernández, M.T. (5) y Jiménez Contreras, E. (5).

A mayor abundamiento, el estudio de Moya y Herrero Solana ${ }^{21}$ sobre visibilidad internacional de la comunidad iberoamericana examina los trabajos de autores iberoamericanos publicados en revistas analizadas por el ISI. D e acuerdo con la presencia en estas revistas, en nuestra área España ocupa el mayor porcentaje de trabajos (48,3 \%) seguida de Brasil (15,3\%), México (11,5\%), Chile (6,2\%) y Portugal (6,2 $\%$ ) aunque en el ámbito internacional USA ocupa el primer lugar con el $58 \%$ de tra bajos, seguidos por el Reino Unido (3,8 \%), Canadá (1,8), Alemania (1,1\%), Bélgica $(0,22 \%$ ), Italia (0,19 \%), Japón (0,18 \%) y España (0,17 \%, 130 trabajos).

La producción por instituciones se observa en el siguiente cuadro:

\begin{tabular}{|l|c|c|}
\hline & $\begin{array}{c}\text { No de } \\
\text { trabajos }\end{array}$ & \% \\
\hline Consejo Superior de Investigaciones Científicas & 33 & 20.2 \\
\hline Universidad Nacional Autónoma de México & 25 & 15.3 \\
\hline Universidad de G ranada & 19 & 11.7 \\
\hline Universidad Carlos III & 17 & 10.4 \\
\hline Universidad de Extremadura & 10 & 6.1 \\
\hline Universidad de Alcalá & 10 & 6.1 \\
\hline Universidad Federal de Río de Janeiro & 9 & 5.5 \\
\hline
\end{tabular}

La repercusión de los trabajos se lleva a cabo mediante la publicación en revistas con el siguiente porcentaje de productividad:

21 Moya Anegón, Félix de y Herrero Solana, Víctor. "Visibilidad internacional..." 


\begin{tabular}{|l|c|c|}
\hline & $\begin{array}{c}\text { No de } \\
\text { trabajos }\end{array}$ & \% \\
\hline Saientomenics & 87 & 34.7 \\
\hline Jarmal of Information Saience & 16 & 6.4 \\
\hline Interienia & 13 & 5.2 \\
\hline Jarmal ofASIS & 12 & 4.8 \\
\hline IFLA Jaumal & 10 & 4 \\
\hline Intem Fonmmi.S. & 9 & 3.6 \\
\hline
\end{tabular}

\section{CONSIDERACIONES FIN ALES}

1. El estudio de los problemas relacionados con el diagnóstico y desarrollo de la investigación documental en España está adquiriendo un franco progreso. D ebe destacarse la reciente aparición del libro de conjunto LainvectigacónenBiblidecono máa Documentacoón(Gijón, Trea, 2002) debido a la pluma del profesor Emilio Delgado López-Cózar, de la Universidad de Granada.

2. Las tesis doctorales en nuestro campo constituyen una de las manifestaciones más importantes dado su necesario componente deinnovación y originalidad. Junto a otras publicaciones científicas, debemos esforzarnos en buscar la calidad a partir, al menos, de entender por investigación aquellos trabajos que tratan de resolver problemas científicos de nuestro campo y, por consiguiente, suponen una verdadera aportación.

3. La formación de investigadores se lleva a cabo a través de los medios habituales: asignaturas adhoc, cursos de doctorado y, fundamentalmente, la labor del director 0 asesor de investigación. En este sentido, nos parece conveniente la redacción de sendos manuales de metodología de la investigación en ByD y de dirección de tesis doctorales, así como la intensificación de los cursos de metodología preceptivos en los programas de doctorado en las universidades.

4. Las tendencias y repercusiones de la investigación en España pueden ser examinadas desde el punto de vista de la realización y difusión de las tesis doctorales. Así, cabe establer conceptos como foco de investigación, escuelas científicas, ge nealogías de directores e influencia de unas universidades sobre otras.

5. La vinculación cada vez mayor entre investigadores universitarios e investigadores profesionales se revela como un mecanismo que permite potenciar y aprovechar al máximo el esfuerzo investigador. 
6. La Base de D atos de Citas (1985-mayo 2002) de autor dirigida por el profesor Fé lix de Moya, catedrático de la Universidad de Granada, permite establecer corrientes de repercusión y propagación de las ideas científicas a nivel deautores españoles y extranjeros más citados, de departamentos universitarios que reciben más citas (Universidad Complutense de Madrid), de departamentos universitarios con más investigadores (Universidad de Granada).

7. La repercusión de la investigación extranjera es nuestro país es todavía muy grande en el contexto de la lista de autores que han recibido más citas. Así, resulta que el porcentaje de autores extranjeros (50\%) recibe el 40\% de las citas. Sin embargo, la tendencia se invierte y el núcleo español es mucho más importante si el examen se restringe a la lista de autores con 50 citas: el 58\% de autores españoles recibe el $62 \%$ de citas.

8. La repercusión de la investigación española o iberoamericana en el ámbito de la ciencia mundial es todavía relativamente pequeña. Los datos de que disponemos indican que España es el país que más produce (48,3 \%) seguido de Brasil (15,3 $\%$ ), México (11,5\%), Chile (6,2\% ) y Portugal (6,2\%). En relación con el resto de países, España ha producido 130 trabajos, lo que representa el 0,17 \% de la producción mundial registrada en revistas contenidas en los repertorios ISI.

10. La aportación investigadora de los profesionales de la biblioteca y documentación arroja un 30\% aproximadamente, tanto en número de autores como de citas recibidas, frente al grupo académico (Universidad yCSIC) que registraun $20 \%$ de autores y un 30\% de citas. Sin embargo, en la lista de autores citados con 50 citas al menos, el grupo académico domina la situación: 58\% y $62 \%$ de autores y citas respectivamente, frente a $21 \%$ y $16 \%$ de citas de autoresy citas respectivamente.

\section{BIBLIOGRAFÍA}

Arquero Avilés, Rosario. Laproduciónäentífica enBibidteconmáayo amentadónenEspaña(1975-1984). Tesis doctoral. Madrid, Universidad Complutense de Madrid, Facultad de Ciencias de la Información, 2001.

Borrego Huerta, Ángel. "La investigación cualitativay sus aplicaciones en Biblioteconomía y D ocumentación", en ReistaEspeñdade Dcamentacón Científica, 22, 2, 1999, pp. 139-156.

- - - . Metoddoǵa aralitativa deinvestigaaónenBiblicteconomáay Dœumentaaón Tesis doctoral dir. por J.A. Frías. Salamanca, Universidad de Salamanca, 2001, inédita.

Cano, Virginia. "Bibliometric Overview of Library and Information Science Research in Spain", en Jaumal of theAmeican Sociey for Information Saience, vol. 50, 끙, 1999, pp. 675-680. 
D elgado, Juan Manuel y Gutiérrez, Juan (Coor.). Méodbsyténicasara litativasdeinvestigaiónenCieniassociales Madrid, Síntesis, 1995, 669 págs.

D elgado López-Cózar, Emilio. "Diagnóstico de la investigación en Biblioteconomía y D ocumentación en España" (1976-1996), en Jarmal of Spanish Restrch on Information Säence, I, 1, January-June 2000, pp.

- - -. “La investigación en Biblioteconomía y D ocumentación” Gijón, Trea, 2002, 254 págs.

- - -. "Las revistas españolas deCiencias de la D ocumentación, productos manifiestamente mejorables", en El Profesional dela Informacón 10, 12, diciembre de 2001. Pp. 46-56.

D esantes G uanter, José M. y López Yepes, José. Teenay ténicadela investigacoón dentífica Madrid, Síntesis, 1996, 268 págs.

Frías, J. A y Romero, P. “ ¿Q uienes son y quécitan los investigadores que publican en las revistas españolas de Biblioteconomía y D ocumentación?", en Anales deDcumentacón 1, 1998, pp. 29-53.

Gómez Caridad, I.; Sancho, Rosa y Moreno, Luz y Fernández, M. Teresa. "Influence of Latin A merican Journals coverage by international databases", en Scientomerics 46, 3, 1999, pp. 443-456.

Jiménez Contreras, Evaristo y Moya Anegón, Félix de. "Análisis de la autoría en revistas españolas de Biblioteconomíay D ocumentación” , 1975-1995, en ReistaEspeñdadeDoumentaciónCientífica, 20 (3), 1997, pp. 252-267.

López Gijón, J.A.; Pérez López, A. y Ruiz de Villegas, M. “Siete Jornadas Bibliotecarias de Andalucia", en VII JomadasBibidtecaniasde Andaluáa Huelva, Diputación Provincial, 1995, pp. 229-246.

López López, Pedro. "Bibliometría: La medida de la información", en López Yepes, José (Coor.). Manual deInfomacoóny Docmentaaón Madrid, Pirámide, 1996, pp. 488-512.

López Piñero, José María y Terrada, María Luz. "Los indicadores bibliométricos y la evaluación de la actividad médico-científica", en MedianaClínica, 98, 1992, pp. 64-68, 101-106, 142-148y 384-388.

López Y epes, José. “Focos de investigación y escuelas científicas en D ocumentación . La experiencia de las tesis doctorales", en Profe sional dela Infomacón 11, 1, enero-febrero 2002, pp. 46-52.

- - - Laaventura delainvestigacóndentífica Gúa da investigadorydd diretor deinvetigaión Madrid, Síntesis, 1995, 253 págs. 
López Y epes, José. Loscaminosdelainfomadón Cómbluscar, sdecianar yorganizar nuestra docamentaaón pessonal. Madrid, Fragua, 1997, 345 págs.

- - -. "La evaluación de la ciencia en el contexto de las Ciencias de la D ocumentación", en InvesigadónBibidteedógia, vol. 13, nํ27, julio-diciembre 1999, pp. 195-212.

Merlo Vega, José A ntonio y Sorli Rojo, Angela. BiblideenomáayDœr mentacoónen Intemet Madrid, Cindoc, C.S.I.C., 1997, 150 págs.

Moya Anegón, Félix de; López Gijón, J. y García Caro, C. Ténicas arantitativas aplicadasa la Biblidteconmáa yla Doumentaión Madrid, Síntesis, 1996.

- - - y Jiménez Contreras, E. ResarchFrontsinLibraryandInfomation Saience in Spain (1985-1994). "Scientometrics", 42,2, 1998, pp. 229-246.

- - - y Jiménez Contreras, Evaristo. "Autores españoles más citados en Biblioteconomía y D ocumentación", en El profesional dela infomacón, vol. 8, oㅡ 5, mayo 1999, pp. 28-29.

- - - y Herrero Solana, Víctor. "Visibilidad internacional de la producción científica iberoamericana en Biblioteconomía y D ocumentación" (1991-1999), en V Enautro EDIBCIT. Granada, Universidad de G ranada, 2000, pp. 341-370.

- - - . "La investigación española en Recuperación de la Información: A nálisis bibliométrico (1984-1999)", en Jaumal ofSpanishRe serch on Information Sáence, I, 1, January-June 2000, pp. 117-123.

Ríos Hilario, Ana Belén. "Metodologías, técnicas y estrategias de investigación en las Jomadas Españolas de D ocumentación Automatizada (1981-1996)" , en VI JomadasEspañdas deDcumentacoón, Valencia, 1998, pp. Jornadas Fesabid. Valencia, 1998, II, pp. 735743

Tramullas Saz, Jesús (Ed.). Tendenias dela investigacón en Doamentar dớ Zaragoza, Universidad de Zaragoza, 1996, 259 págs. 